\title{
Industrias de fundición: aspectos ambientales e indicadores de condición ambiental $\left.\right|^{(\bullet)}$
}

\author{
B.S. Sosa*, R.B. Banda-Noriega* y E.M. Guerrero* \\ Resumen \\ El empleo de indicadores ambientales es, actualmente, una herramienta que colabora en el proceso de toma de \\ decisiones en la gestión de la administración pública y privada. La falta de antecedentes de Indicadores de Condición \\ Ambiental (ICA) en lo regional y local, la información asociada a la generación de residuos, efluentes y emisiones \\ gaseosas provenientes de la industria de fundición, y la particularidad de encontrar a estas industrias en el ámbito \\ urbano en la ciudad de Tandil, Argentina, orientan el principal objetivo del presente trabajo: la construcción de un \\ conjunto de ICA que proporcionen información sobre el estado del ambiente en relación a este tipo de actividad. \\ El campo de la investigación comprendió el total de industrias de fundición de la ciudad en el periodo marzo - abril \\ de 2010. Se generaron ICA para el recurso, aire (9), suelo (5) y agua (1) con metodología adaptada a cada caso par- \\ ticular.
}

Palabras clave Indicadores de Condición Ambiental (ICA); Emisiones; Contaminación ambiental urbana; Tandil; Argentina; Gestión ambiental.

\section{Foundry industries: environmental aspects and environmental condition indicators}

\begin{abstract}
Nowadays, environmental indicators are widely used as effective tools to assist decision-making in both public and private sectors. The lack of literature and research about local and regional Environmental Condition Indicators $(\mathrm{ECI})$, the poor knowledge regarding solid waste generation, effluents and gas emissions from foundry industries, and their particular location in the urban area of Tandil, Argentina are the main reasons for this investigation, aiming to develop a set a of ECI to provide information about the environment in relation to the foundry industry. The study involves all the foundries located in the city between March and April 2010. The set of ECI developed includes 9 indicators for air, 5 for soil and 1 for water. Specific methodology was used for each indicator.
\end{abstract}

Keywords

Environmental Condition Indicators (ECI); Emissions; Urban environmental pollution; Tandil; Argentina; Environmental management.

\section{INTRODUCCIÓN}

El rápido crecimiento económico e industrial de las últimas décadas ha traído consigo serios problemas de contaminación ambiental, como la polución del aire, agua y suelo. Paralelamente las exigencias y controles respecto de la relación actividad / entorno, por organismos de fiscalización, ha propiciado la creación de herramientas que sirven a la evaluación del estado del ambiente. Los indicadores ambientales son ejemplo de ello.
Los antecedentes en utilización de indicadores de condición ambiental (ICA) se corresponden, en general, con empresas de gran magnitud, que poseen sistema de gestión medioambiental (SGMA), y evaluación del desempeño ambiental de la empresa de acuerdo con la ISO 14031. La ISO 14032 ejemplifica el desarrollo de ICA a nivel internacional (cítese el caso de Iran Khodro Co, fábricas de automóviles iraníes), donde se encuentran empresas radicadas en Argentina, cítese el caso de Envases Alvher, División Industrial de Dinan

(•) Trabajo recibido el día 20 de diciembre de 2011 y aceptado en su forma final el día 20 de septiembre de 2012.

* CICPBA. Centro de Investigaciones y Estudios Ambientales (CINEA, FCH-UNICEN). E-mail: beatrizsosa33@gmail.com. 
S.A, envases flexibles laminados, YPF Refinería Lujan de Cuyo, una refinería de una petrolera multinacional y Petroquímica Cuyo S. A. I. C., una petroquímica ${ }^{[1]}$.

En referencia a industrias que son específicas de fundición (mucho menos en la escala que se considera Pyme) no se hallan antecedentes de ICA aunque sí de indicadores de sustentabilidad ambiental. El trabajo "Worldsteel Sustainability Indicator Methodogy" define y calcula una metodología para indicadores de sustentabilidad de las industrias del Hierro y el Acero $^{[2]}$. En lo nacional, la empresa Acindar Grupo Arcelor Mital, en su planta de Villa Constitución tiene desarrollado el índice de desempeño ambiental donde, mediante indicadores de gestión ambiental, se controlan emisiones a la atmósfera, se evalúa la contaminación de los efluentes líquidos y la generación de residuos ${ }^{[3]}$.

En cuanto a las condiciones del estado del ambiente la información disponible se encuentra referida como "reportes del estado del ambiente" en cuestiones que se relacionan con problemáticas globales e indicadores de seguimiento (ej. concentración de gases de efecto invernadero), pero no están asociados y/o determinados por una actividad en particular (véase p. ej. la Iniciativa Latinoamericana y Caribeña para el Desarrollo Sostenible ${ }^{[4]}$; GEO América Latina y el Caribe ${ }^{[5]}$; Primer Compendio de Estadísticas Ambientales, República Argentina ${ }^{[6]}$ ).

Si bien la relación empresa/entorno tiene características propias de cada lugar donde ésta se desarrolla, hay aspectos ambientales que se pueden recuperar y generalizar para una actividad en particular. Es de interés por tanto desarrollar herramientas capaces de prevenir problemas ambientales consecuentes de la interacción elemento de la actividad/ entorno. La dificultad de acceso a antecedentes de investigación que desarrollen indicadores de desempeño ambiental en industrias de fundición, y más aún la ausencia de los ICA que forman parte de este grupo, es lo que da motivo a su construcción en esta investigación, y constituye el objetivo del presente trabajo. Se espera entonces que estas herramientas permitan hacer el seguimiento y control del estado del ambiente en el entorno de las fundiciones.

Se adelanta brevemente que (hay un apartado metodológico específico para ello) las decisiones metodológicas que se llevan a cabo para la selección de los ICA se basan en criterios de relevancia, es decir, de pertinencia del indicador para los problemas o decisiones en que se requiere el uso y la relación que éstos tienen con normas específicas que existen en el país o en otras escalas territoriales, lo que lo sitúa en la realidad en términos de políticas públicas e información ciudadana.

\section{DESCRIPCIÓN DE LA ZONA DE ESTUDIO}

El proceso de industrialización desde el punto de vista espacial en la Argentina, se produjo de forma diferencial y en relación con ciertos factores de localización como los medios de transporte, la localización de la materia prima, el mercado, las fuentes de energía, la infraestructura y por supuesto, las facilidades impositivas de cada lugar ${ }^{[7]}$.

En la ciudad de Tandil, a partir de la década de 1920 y hasta la primera década del siglo XXI, las industrias metalúrgicas y en específico las de fundición han sido motores de crecimiento económico y generación de empleo ${ }^{[8]}$. Este proceso paralelo de desarrollo industrial y urbano, algo desordenado, ha generado que con el paso del tiempo las industrias quedaran inmersas en zonas urbanas y periurbanas (Fig. 1).

Según normativa local y provincial vigente (Ordenanza Municipal № 9865/05, Decreto Municipal № 3085/07, Decreto Ley Provincial № 11.459/93, Decreto Provincial № 8912/77, Decreto Reglamentario Provincial № 1741/96), las industrias de fundición están localizadas en las Zonas de Regulación General. Es decir, se ubican en sectores con características homogéneas en cuanto a sus aspectos socio-económicos, paisajísticos y ambientales, en relación a los usos y ocupación del suelo, en lo referido al patrimonio urbano-arquitectónico y, en particular, en el rol que cumplen en la estructuración general y en la dinámica funcional del espacio territorial de Tandil. En dicha zona no está permitida la instalación de industrias de segunda (Ley 11.459, Art 15 inciso "b") y tercera (Ley 11.459, Art 15 inciso "c") categoría, a pesar de que las industrias de fundición, por las características que reúnen, pertenecen a estas categorías ${ }^{[8]}$.

Estudios previos describen las fundiciones según sus condiciones productivas, operativas y ambientales. De las 30 industrias existentes, el $80 \%$ se dedican a la producción de piezas de un solo tipo de material. El $20 \%$ restante se distribuye entre fundiciones de $\mathrm{Al} / \mathrm{Fe}$, $\mathrm{AL} / \mathrm{Fe} / \mathrm{Cu}+\mathrm{Sn}$ (bronce), y $\mathrm{Al} / \mathrm{Cu}+\mathrm{Sn}$. Del $80 \%$, el $60 \%$ se dedica al hierro y sus aleaciones, y el $40 \%$ al aluminio. De acuerdo a estimaciones correspondientes al año 2010, la producción en toneladas ( $\mathrm{t}$ ) de piezas fundidas en esta localidad asciende a $3.011 \mathrm{t}$ por mes, de las cuales 2.978,5 t corresponden a hierro gris y aleaciones de hierro, 30,5 t constituyen aluminio y $2 \mathrm{t}$ pertenecen a bronce ${ }^{[9]}$; es de destacar que sólo una industria concentra el $80 \%$ de la producción mensual.

Respecto de las condiciones operativas, a partir de la clasificación de la OCDE del año 2001, que atiende las intensidades de I+D (se calcula a partir de dos medidas de la producción: valor de producción y valor agregado), y de las denominaciones y códigos ISIC Rev 3 


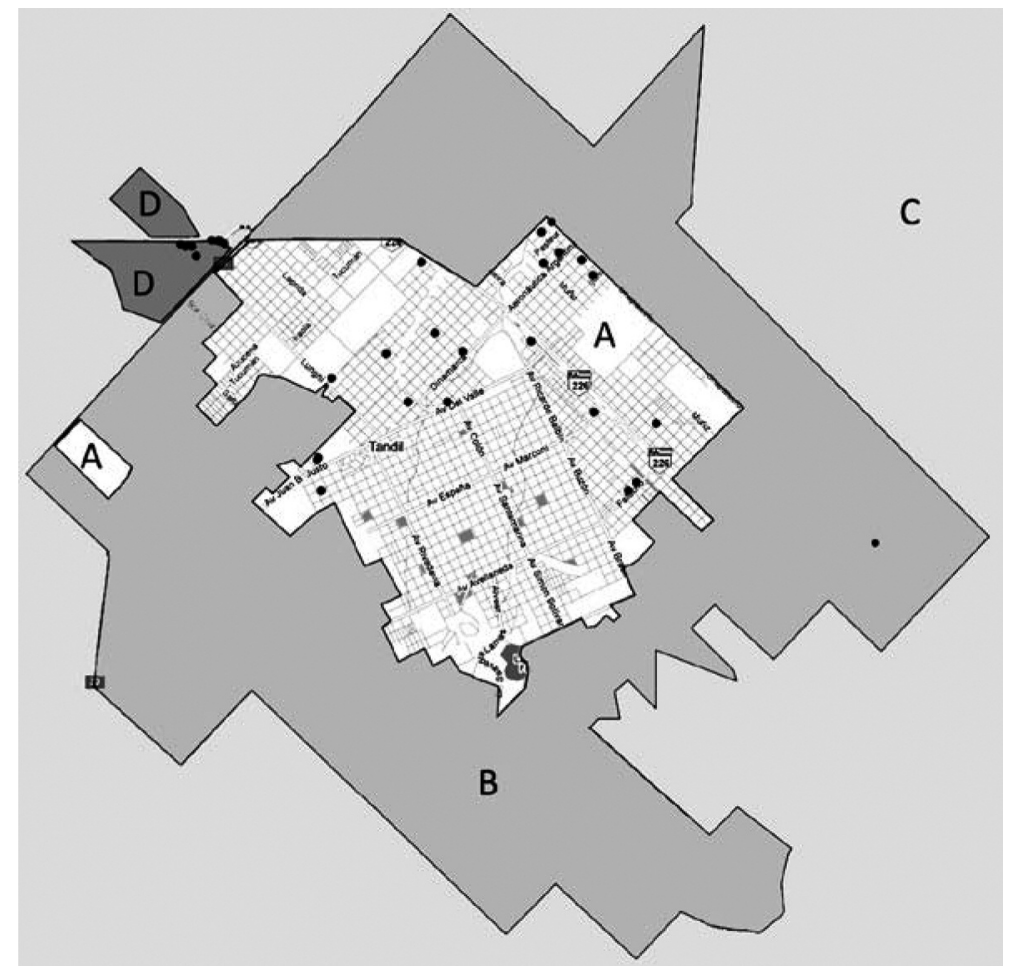

Figura 1. Distribución de industrias de fundición. Los puntos representan las industrias. Los polígonos indican: $(A)$ área urbana, $(B)$ complementaria, (C) rural y (D) zona de uso industrial.

Figure 1. Distribution of foundry industries. Points represent industries. Polygons represent: (A) urban areas, $(B)$ complementary areas, $(C)$ rural areas and (D) near industrial use.

(International Standard Industrial Classification), Argentina identifica a la industria manufactura de metales básicos como de media baja tecnología ${ }^{[10]}$. Estudios locales indican además, que estas industrias son en su mayoría pequeñas en tamaño (de acuerdo con la cantidad de empleados por empresa), siendo las condiciones operativas y de cumplimiento de la legislación vigente preocupantes en relación al control de los efluentes ${ }^{[9]}$.

En este sentido las condiciones ambientales señalan problemas como falta de chimeneas o de tratamientos de efluentes gaseosos que se agravan con el uso, aún significativo, de hornos cubilotes en empresas que funden hierro gris. Los hornos que funden aluminio (crisol y reverbero) en general poseen campanas de extracción de gases, sin tratamiento y con chimeneas de baja altura. En esta actividad, sólo se cuenta con chimeneas para evacuar los gases captados en el ambiente laboral, desatendiendo las obligaciones normativas (Tabla I).

A excepción de los efluentes sanitarios, el volumen de aguas residuales industriales de las fundiciones

Tabla I. Sistemas de tratamiento de efluentes gaseosos en hornos de fusión correspondientes a las 30 industrias locales

Table I. Emission control systems in furnaces of the 30 local foundries

\begin{tabular}{lcccc}
\hline Horno de fusión & Cubilote & Eléctrico & Crisol & Reverbero \\
\hline $\begin{array}{l}\text { Hornos totales } \\
\text { Hornos con ausencia de }\end{array}$ & 13 & 12 & 14 & 3 \\
tratamiento de efluentes gaseosos & 6 & 5 & 14 & 3 \\
\hline
\end{tabular}


es poco significativo. El principal problema puede darse por el contacto del agua de escorrentía con materias primas de fundición, escorias, lodos, cenizas y otros residuos ${ }^{[11]}$. Veintiuna industrias vierten los efluentes líquidos del proceso al sistema de alcantarillado sanitario. Dos quedan por fuera de este servicio y disponen las aguas residuales en pozos absorbentes, y otras siete restantes se radican en el Parque Industrial Tandil (PIT) cuya infraestructura cuenta con un canal de desagüe (del año 1970) que sirve a todas las empresas en el parque y descarga aguas abajo en el arroyo Langueyú. No obstante, como ya se mencionara, la gran mayoría de estas industrias se encuentra en zonas con sistema de alcantarillado por lo que no representaría un problema ambiental significativo si se considera que las aguas colectadas por dicho sistema son posteriormente tratadas.

De acuerdo con lo expuesto se hace necesario abordar las situaciones de incompatibilidad de usos de suelo y problemáticas ambientales propias de las emisiones que la actividad genera.

\section{PROCESOS Y ASPECTOS AMBIENTALES TÍPICOS EN INDUSTRIAS DE FUNDICIÓN}

Las industrias de fundición son establecimientos que obtienen como resultado de su proceso piezas de metal que no podrían ser producidas por procesos de laminación forja o soldadura.

Los procesos que se llevan a cabo en cada fundición presentan variantes que dependen del tipo de metal a fundir (fundición de metales ferrosos como el hierro gris, hierro nodular, aceros y no ferrosos como el aluminio, bronce, cobre, zinc, plomo y níquel entre otros), de los métodos y de las tecnologías aplicadas.

Los diferentes métodos para la obtención de las piezas dependerán del tipo de pieza y cantidad a producir. Las etapas en general involucran: manipulación y almacenamiento de materiales, fabricación de moldes y noyos, fusión del metal, colada y limpieza de piezas fundidas. A continuación (Tabla II) se presentan las etapas que se desarrollan en el ámbito de la fundición y los aspectos ambientales asociados. Se entiende por aspecto ambiental a aquel elemento de la actividad capaz de interactuar con el entorno y producir un impacto ${ }^{[12]}$.

De acuerdo a los aspectos ambientales identificados en las distintas etapas del proceso, los ICA se relacionarían primordialmente con las emisiones gaseosas (material particulado y otros contaminantes atmosféricos) y con residuos sólidos, fundamentalmente arenas de descarte y escorias. Teniendo en cuenta factores tales como las tecnologías actuales de los hornos de fundición que superan las viejas tecnologías que tenían un consumo significativo de agua, asimismo el tamaño, mediano a pequeño, de las industrias de fundición de la zona de estudio, el acceso de la mayoría al sistema de alcantarillado sanitario y la generación de volúmenes poco significativos de efluentes líquidos (ver apartado 2), se considera a estos efluentes como un aspecto poco relevante para la actividad. Sin embargo, el recurso hídrico será igualmente abordado ya que se identifica un riesgo de contaminación al recurso hídrico subterráneo debido a las disposiciones de residuos de arenas de fundición en cavas originadas por la extracción de granito y actualmente abandonadas, características de esta zona de estudio.

Otros aspectos ambientales a tener en cuenta en esta actividad, con carácter secundario, son la generación de emisiones difusas, olores y humos que causan molestias de diversa índole (olor durante las horas de fusión, degradación de las fachadas de las construciones vecinas por humos y emisión de material particulado, en especial por fundición de hierro gris en hornos cubilotes) y el ruido proveniente del funcionamiento de la fábrica, de la movilidad de vehículos de carga y descarga de materiales, residuos y piezas fundidas.

\section{METODOLOGÍA}

El campo bajo investigación comprendió el total de industrias de fundición de la ciudad de Tandil, involucrando 30 establecimientos identificados durante el relevamiento realizado en el periodo marzo - abril de 2010. Debe destacarse aquí, que existe la posibilidad de que establecimientos no declarados funcionen en fondos de viviendas o galpones sin autorización.

El conjunto sometido a análisis quedó definido por los cinco tipos de procesos productivos existentes en el área de estudio que corresponden a los siguientes metales: aluminio $(\mathrm{Al})$, aluminio/hierro $(\mathrm{Al} / \mathrm{Fe})$, aluminio/bronce $(\mathrm{Al} / \mathrm{Cu}+\mathrm{Sn})$, aluminio/hierro/bronce $(\mathrm{Al} / \mathrm{Fe} / \mathrm{Cu}+\mathrm{Sn})$, hierro $(\mathrm{Fe})$.

Los pasos metodológicos seguidos en la construcción de los indicadores están tomados y adaptados de la metodología propuesta por Jane Barr $(2006)^{[8 \text { y } 15]}$, donde: a) se identifican los puntos de interacción entre el entorno y la actividad, para lo cual se hizo una revisión bibliográfica obteniendo los aspectos ambientales recopilados en la tabla II; b) se describen los factores ambientales afectados, se identificaron temas prioritarios en relación a problemáticas ambientales locales y la actividad en 
INDUSTRIAS DE FUNDICIÓN: ASPECTOS AMBIENTALES E INDICADORES DE CONDICIÓN AMBIENTAL FOUNDRY INDUSTRIES: ENVIRONMENTAL ASPECTS AND ENVIRONMENTAL CONDITION INDICATORS

Tabla II. Aspectos ambientales asociados a cada etapa del proceso

Table II. Environmental aspects related to each process stage

\begin{tabular}{|c|c|c|c|}
\hline $\begin{array}{l}\text { Etapa } \\
\text { del proceso }\end{array}$ & $\begin{array}{l}\text { Recurso } \\
\text { con } \\
\text { potencial } \\
\text { impacto }\end{array}$ & Aspecto ambiental asociado & Efluente \\
\hline \multirow{3}{*}{$\begin{array}{l}\text { Manipulación y } \\
\text { almacenamiento } \\
\text { de materiales e } \\
\text { insumos }\end{array}$} & Aire & \multirow{3}{*}{$\begin{array}{l}\text { Descarga, almacenaje y } \\
\text { transporte de materias primas } \\
\text { (metales, chatarra) e insumos } \\
\text { (arenas, combustibles sólidos y } \\
\text { líquidos, resinas, fundentes, } \\
\text { solventes, etc.). }\end{array}$} & $\begin{array}{l}\text { Material particulado, hidrocarburos, } \\
\mathrm{COV}, \mathrm{Cu}, \mathrm{Ni}, \mathrm{Zn}, \mathrm{C} \text {. }\end{array}$ \\
\hline & Agua & & $\begin{array}{l}\text { Aguas residuales con solventes, } \\
\text { ácidos varios, metales, fenoles. }\end{array}$ \\
\hline & Suelo & & $\begin{array}{l}\text { Residuos industriales sólidos: } \\
\text { arenas, restos de combustibles, } \\
\text { bidones. }\end{array}$ \\
\hline \multirow[t]{3}{*}{$\begin{array}{l}\text { Producción de } \\
\text { moldes y noyos }\end{array}$} & Aire & Manipulación de materias primas. & $\begin{array}{l}\text { Material particulado. Metales. } \\
\text { Compuestos orgánicos volátiles } \\
(\text { COV). }\end{array}$ \\
\hline & Agua & $\begin{array}{l}\text { Lavado de cajas de noyos y } \\
\text { bateas de pinturas de sección } \\
\text { noyería y rebabado. Lodos. }\end{array}$ & $\begin{array}{l}\text { Kerosene, gasoil, soda } \\
\text { caústica, pinturas y restos de } \\
\text { pinturas. Lodos con contenidos de } \\
\text { metales. }\end{array}$ \\
\hline & Suelo & Manipulación de materias primas. & $\begin{array}{l}\text { Polvo y barridos con contenidos } \\
\text { de metales. }\end{array}$ \\
\hline \multirow[t]{3}{*}{$\begin{array}{l}\text { Proceso de } \\
\text { fusión }\end{array}$} & Aire & $\begin{array}{l}\text { Emisiones controladas: focos } \\
\text { estacionarios con sistemas } \\
\text { tradicionales de limpieza de gases. } \\
\text { Emisiones fugitivas: aperturas de } \\
\text { tapas o puertas para cargar, } \\
\text { recargar, alear, inyectar oxígeno, } \\
\text { remover la escoria y al colar. }\end{array}$ & $\begin{array}{l}\text { Material particulado, metales, } \\
\text { monóxido de carbono (CO), } \\
\text { compuestos orgánicos volátiles } \\
\text { (COV), óxidos de azufre, óxidos de } \\
\text { nitrógeno, humos inorgánicos, } \\
\text { dioxinas. }\end{array}$ \\
\hline & Agua & $\begin{array}{l}\text { Sistemas de tratamientos de } \\
\text { efluentes gaseosos (ej. lavador de } \\
\text { gases). }\end{array}$ & $\begin{array}{l}\text { Carbonilla, óxidos de } \\
\text { manganeso, óxidos de hierro y } \\
\text { otras impurezas. Carga térmica } \\
\text { importante. Metales. }\end{array}$ \\
\hline & Suelo & $\begin{array}{l}\text { Sistema de tratamiento de } \\
\text { efluentes gaseosos. }\end{array}$ & Metales en polvo y lodos de filtros. \\
\hline \multirow[t]{3}{*}{$\begin{array}{l}\text { Colada y } \\
\text { enfriamiento }\end{array}$} & Aire & Llenado de moldes y enfriamiento. & $\begin{array}{l}\text { Material particulado. Monóxido de } \\
\text { carbono (CO), compuestos } \\
\text { orgánicos volátiles (COV) }\end{array}$ \\
\hline & Agua & $\begin{array}{l}\text { Enfriamiento y arrastre de la } \\
\text { escoria. }\end{array}$ & Metales. \\
\hline & Suelo & Residuos de escorias. & $\begin{array}{l}\text { Escorias de hierro y acero. Escoria } \\
\text { de aluminio, plomo, cobre, estaño, } \\
\text { zinc y aleaciones como zamak, } \\
\text { latón y bronce. Dioxinas. }\end{array}$ \\
\hline
\end{tabular}


Tabla II (continuación). Aspectos ambientales asociados a cada etapa del proceso

Table II (continuation). Environmental aspects related to each process stage

\begin{tabular}{|c|c|c|c|}
\hline $\begin{array}{l}\text { Etapa } \\
\text { del proceso }\end{array}$ & $\begin{array}{l}\text { Recurso } \\
\text { con } \\
\text { potencial } \\
\text { impacto }\end{array}$ & Aspecto ambiental asociado & Efluente \\
\hline \multirow[t]{3}{*}{ Desmoldeo } & Aire & \multirow[b]{3}{*}{ Desmolde } & Material particulado \\
\hline & Agua & & Aceites, solventes, ácidos. \\
\hline & Suelo & & $\begin{array}{l}\text { Arenas de descarte o desechadas } \\
\text { con contenidos de resinas y } \\
\text { catalizadores, pinturas, pastinas, } \\
\text { endurecedores y partículas de } \\
\text { metales fundidos. } \\
\text { Desmoldantes. Elastizantes en } \\
\text { tierra de moldeo. }\end{array}$ \\
\hline \multirow[t]{3}{*}{$\begin{array}{l}\text { Limpieza y } \\
\text { terminación }\end{array}$} & Aire & Limpieza de productos fundidos. & $\begin{array}{l}\text { Material particulado. } \\
\text { Polvo de granalla. }\end{array}$ \\
\hline & Agua & $\begin{array}{l}\text { Limpieza de maquinarias. Limpieza } \\
\text { de las piezas producidas. Tareas } \\
\text { de lavado de material y desechos } \\
\text { de reactivos de laboratorio. }\end{array}$ & $\begin{array}{l}\text { Residuos líquidos: solventes, } \\
\text { ácidos, álcalis. Aguas residuales } \\
\text { con solventes y grasas. } \\
\text { Ácidos varios y otras drogas de } \\
\text { laboratorio. }\end{array}$ \\
\hline & Suelo & Materiales gastados. & $\begin{array}{l}\text { Piedra caliza, material refractario } \\
\text { del horno, ladrillos y arenas de } \\
\text { crisoles. Materiales impregnados en } \\
\text { aceite. }\end{array}$ \\
\hline
\end{tabular}

Fuente: Elaboración propia. Adaptado de Asimet - Consejo Nacional de Producción Limpia[13], Ministerio de Medio Ambiente Documento BREF[14] y ODES[11].

cuestión sobre la base de entrevistas a informantes calificados del Sistema de Gestión Ambiental de la Dirección de Medio Ambiente del Municipio de Tandil, del sector privado, y de bibliografía específica sobre las condiciones naturales del medio, y c) se construyen indicadores de condición para dichos factores $^{[15}$ y 16]. En este último paso, además de cumplir con los criterios comunes a todo indicador (disponibilidad de información estadística de calidad, simplicidad, precisión y claridad, consistencia interna de la hoja metodológica, etc. $)^{[16]}$, se contempla a quién va destinado, cuáles son las fuentes y carencias de datos, y se evalúa su fortaleza y debilidad. Al mismo tiempo, y con motivo de hacer más claro los campos descriptivos que hacen al indicador, se anexaron para su desarrollo campos sugeridos por Quiroga Martínez (2009) ${ }^{[16]}$ : descripción del indicador, periodicidad de los datos (periodo de tiempo en que se actualiza) y la fórmula de cálculo del indicador.

Los indicadores de condición ambiental propuestos son agrupados de acuerdo al recurso con potencial de afectación. Así se tienen tres grupos de ICA para las industrias de fundición de Tandil: ICA para el recurso aire, suelo y agua, según orden de importancia.

El marco analítico para la selección de los indicadores se basa en la importancia del aspecto ambiental identificado en el contexto de las problemáticas ambientales que la actividad genera. Por tanto el criterio de selección aplicado a cada indicador responde a su pertinencia en los problemas o decisiones en que se quiere utilizar dicho indicador y a la relación que tiene con normas específicas que existen en el país o en otras escalas territoriales, lo que lo sitúa en la realidad en términos de políticas 
INDUSTRIAS DE FUNDICIÓN: ASPECTOS AMBIENTALES E INDICADORES DE CONDICIÓN AMBIENTAL FOUNDRY INDUSTRIES: ENVIRONMENTAL ASPECTS AND ENVIRONMENTAL CONDITION INDICATORS

públicas e información ciudadana ${ }^{[16]}$. En este sentido se utilizó una metodología adecuada a cada caso particular, la cual se explica durante el desarrollo del trabajo.

\section{INDICADORES DE CONDICIÓN AMBIENTAL}

A continuación se desarrollan ICA para el recurso aire, suelo y agua.

\subsection{ICA para aspectos ambientales que impactan el recurso aire}

En la construcción de los indicadores a medir se trabajó sobre la base de las Guías Técnicas para la Medición, Estimación y Cálculo de las Emisiones al Aire, IHOBE [17-19]. En estos documentos técnicos se detallan contaminantes provenientes del horno de fusión usando como variables el tipo de horno y la materia prima, sin necesidad de describir la capacidad de los hornos o el tipo de combustibles, entre otros. Este método no considera para la estimación de emisiones la aplicación de sistemas de tratamiento de efluentes gaseosos, contemplando de esta forma la peor situación posible. Por ello, resulta apropiado su aplicación para este estudio donde la mayoría de las industrias no tienen sistemas de tratamiento (ver apartado 2). Se generó una tabla donde se recopilaron los datos relacionados con las características operativas locales, es decir, tipo de horno (hornos cubilotes, eléctricos, reverbero) y materia prima (hierro, aluminio y cobre).

La lista de contaminantes obtenidos se comparó con los considerados en las normas nacionales (Ley Provincial $N^{\circ}$ 5.965, Decreto Reglamentario $\mathrm{N}^{\circ} 3.395 / 96$, Resolución $\mathrm{N}^{\circ}$ 242/97 [20]) e internacionales (National Ambient Air Quality Standards (NAAQS) ${ }^{[21]}$ y Guías de Calidad de Aire de la OMS $2005^{[22]}$ ) que consideran criterios de salud humana y el cuidado de los bienes materiales.

La creación de los niveles guías de la calidad de aire ambiente, y de los niveles guías de emisión, es decir, de los límites legales (obligatorios y sugeridos) correspondientes a contaminantes atmosféricos se fundamentan en estos criterios.

Durante el análisis se consideraron tanto contaminantes primarios -aquellos emitidos por una fuente directamente a la atmósfera sin sufrir transformación química desde el momento que salen de su punto de emisión- $\left(\mathrm{SO}_{2}, \mathrm{NO}_{\mathrm{x}}, \mathrm{CO}\right.$, etc. $)$, como contaminantes secundarios, es decir una vez que han evolucionado en el medio y han reaccionado química o fotoquímicamente (formaldehido).

En este contexto se proponen ICA para el caso de inmisión de efluentes gaseosos de las fundiciones (Tabla III). La periodicidad propuesta surge de los antecedentes vinculados a los programas de monitoreo y control de las industrias locales de fundición aprobados por el organismo de aplicación (OPDS) en el marco de la legislación provincial vigente:

-Ley 5965/58 de protección a las fuentes de provisión, cursos y cuerpos receptores de agua y de la atmósfera; Decretos reglamentarios 3395/96; Resolución № 242/97; Complementario Decreto 3395/96; Resolución № 279/96; Presentación de la Declaración Jurada de Efluentes Gaseosos Industriales-.

\subsection{ICA para aspectos ambientales que impactan el recurso suelo}

Los principales residuos sólidos generados son las arenas de descarte, escorias, escombros (virutas y chatarras), polvos y arenas retenidos en filtros de mangas. En fundiciones no ferrosas además se pueden generar residuos peligrosos y contaminados con plomo, cobre, níquel y zinc, con frecuencia en elevadas concentraciones totales y extraíbles, provenientes principalmente de la escoria ${ }^{[5]}$.

Las arenas de descarte son, en el proceso en general y en los residuos sólidos en particular, uno de los efluentes más abundantes generados por esta actividad, y por tanto objeto de atención.

Si consideramos las toneladas de arenas de descarte generadas respecto de las toneladas de pieza producida, tenemos que en las aleaciones de hierro y acero la relación es 1:1, mientras que en las de aluminio es $4: 1^{[11]}$. En base a estos datos, actualmente se disponen en algún lugar unas 2.978,5 t de residuos de arenas por mes provenientes de fundiciones ferrosas, y al menos unas $122 \mathrm{t}$ de residuos de arenas provenientes de fundiciones no ferrosas.

En Tandil, el municipio ha prohibido su disposición en el relleno sanitario de la ciudad, aduciendo que existe desconocimiento sobre si se trata de residuos especiales o no, tal como lo establece la Ley Provincial 11720 de Residuos Especiales y su Decreto Reglamentario 806/97 [23]. Actualmente (e históricamente), los residuos de las fundiciones son depositados en cavas de canteras, ladrilleras y terrenos bajos, situados dentro o en cercanías del casco urbano sin ningún tipo de control ${ }^{[24]}$.

Asimismo, un estudio antecedente ha podido localizar un total de 39 sitios relevados, de los cuales 8 


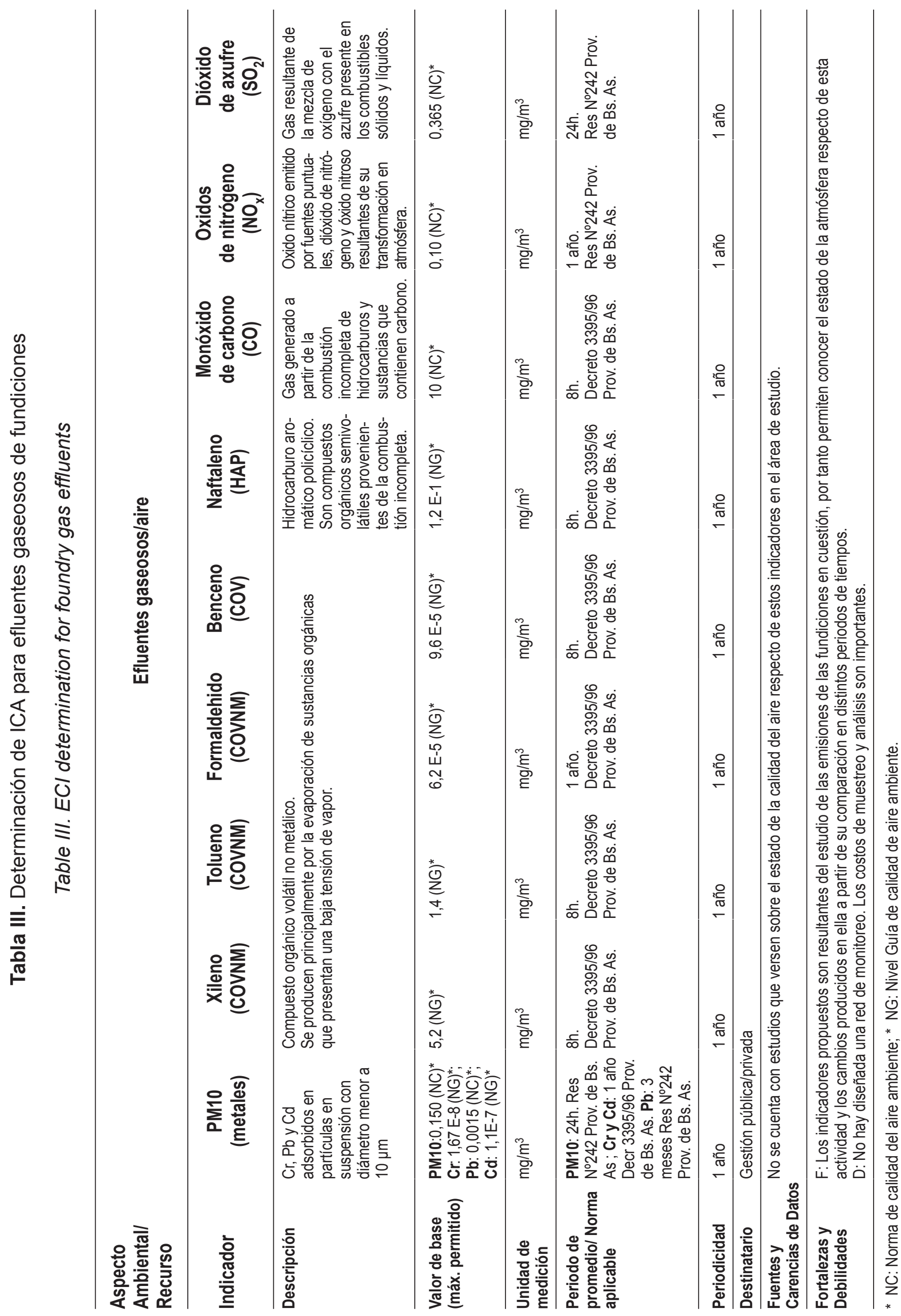


INDUSTRIAS DE FUNDICIÓN: ASPECTOS AMBIENTALES E INDICADORES DE CONDICIÓN AMBIENTAL FOUNDRY INDUSTRIES: ENVIRONMENTAL ASPECTS AND ENVIRONMENTAL CONDITION INDICATORS

pertenecen a disposiciones actuales, 19 a sitios con cese de actividad y 7 a sitios con disposiciones parciales de residuos de arenas de fundición (RAF) ${ }^{[24]}$ (Fig. 2).

Si bien los organismos de control y las industrias de fundición tienden a considerar estos residuos como especiales o peligrosos, gran parte de la literatura disponible expresa que los RAF son mayormente residuos no peligrosos $[25$ y 26]. Antecedentes locales evidencian la necesidad e importancia del monitoreo de fenoles y de metales pesados como el cadmio, cobre, cromo, plomo y zinc debido a la utilización de resinas fenólicas ${ }^{[27]}$. Siempre que los valores de estos elementos estén por debajo de lo estipulado por la legislación

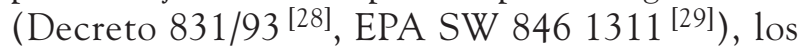
RAF pueden ser considerados como residuos no peligrosos en el marco de la Ley de Residuos Peligrosos 24.051 [28]. Por tanto, se proponen los ICA que figuran en la tabla IV.

En base a lo expuesto, se considera de importancia realizar también el monitoreo de los volúmenes de residuos que se generan en las fundiciones y de los sitios que actualmente son receptores de los mismos. Por tal motivo los ICA para el control de este aspecto ambiental son los que se presentan en la tabla $\mathrm{V}$.
La disposición de estos residuos de arenas representa una amenaza porque la población de Tandil se abastece del acuífero subterráneo local. R. E. Miguel [24], afirma que los variados sectores de disposición final de RAF y otros residuos de fundición constituyen focos multipuntuales que en mayor o menor medida impactan al sistema hídrico subterráneo local. En este sentido, la contaminación del agua del acuífero se produce por el contacto de las arenas con el agua de lluvia cuyo recorrido (proceso de infiltración) termina en la napa freática (lixiviación) ${ }^{[30]}$, significando ello un peligro permanente.

En este contexto, resulta complejo elaborar un indicador que monitoree dicha situación por la falta de disponibilidad de instrumental específico capaz de aportar la información sobre el funcionamiento de este sistema, que contiene múltiples y complejas variables. Si bien este trabajo se limita a construir indicadores aplicables al área de estudio, se considera que de poder contar con estos datos, el indicador debería construirse a partir del concepto de peligro de contaminación del acuífero propuesto por Foster e Hirata (2002) [31] - el uso de la expresión "peligro de contaminación del agua subterránea" tiene exactamente el mismo significado que "riesgo (medido en términos pro-

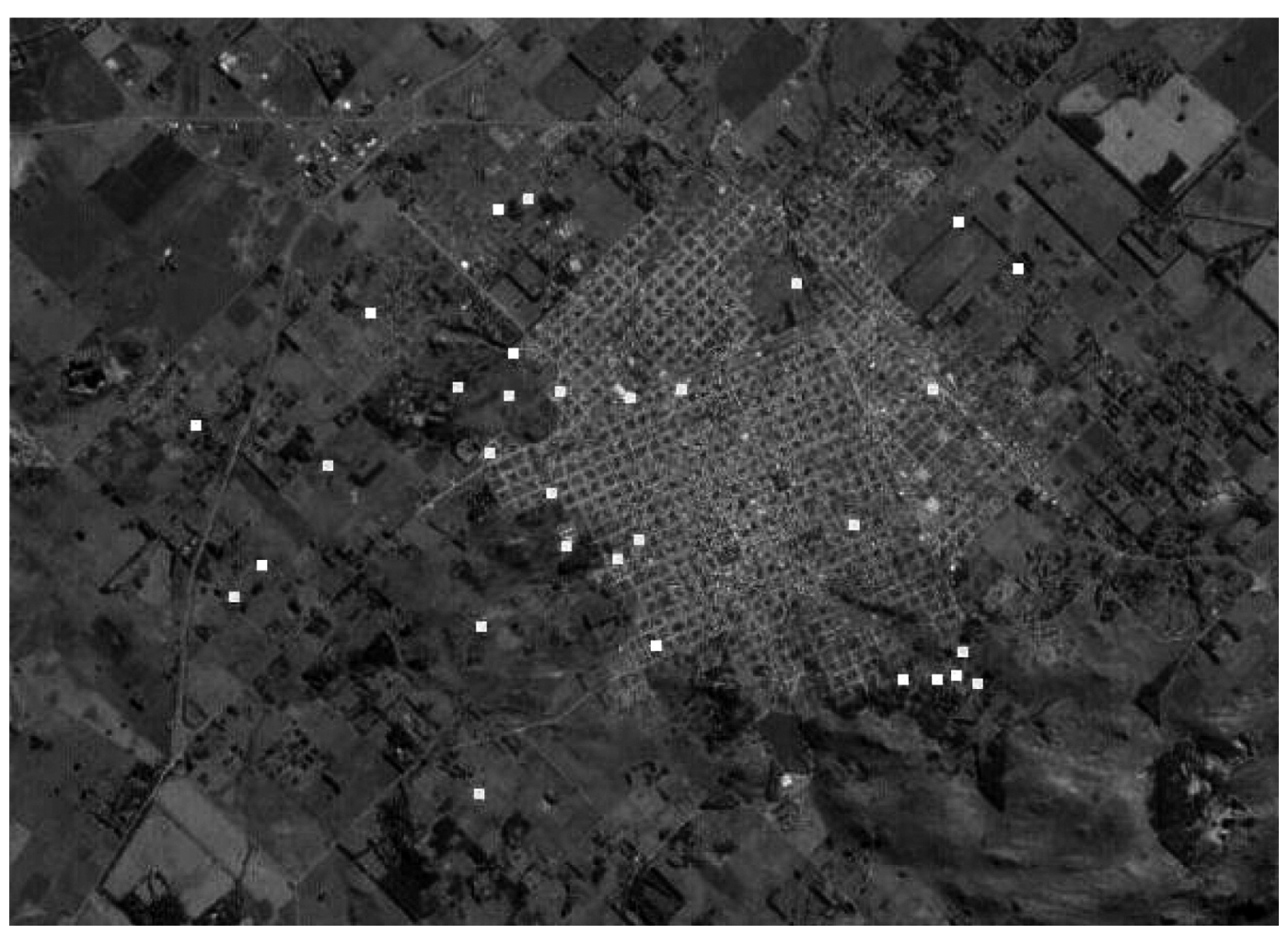

Figura 2. Sitios de disposición final de RAF, extraído de E. Miguel (2009) [24].

Figure 2. Disposal sites for foundry sand wastes, source E. Miguel (2009) [24]. 
Tabla IV. Determinación de ICA para la caracterización química de residuos sólidos de fundiciones

Table IV. ECI determination for chemical characterization of foundry solid wastes

Aspecto ambiental/Recurso

\begin{tabular}{|c|c|c|}
\hline Indicador & Determinación de fenoles & $\begin{array}{l}\text { Determinación de metales } \\
\text { pesados }\end{array}$ \\
\hline Descripción & $\begin{array}{l}\text { Informa sobre la presencia de } \\
\text { fenoles en las arenas }\end{array}$ & $\begin{array}{l}\text { Informa sobre la presencia de } \\
\text { metales pesados }\end{array}$ \\
\hline $\begin{array}{l}\text { Valor de base } \\
\text { (máx. permitido) }\end{array}$ & 2 & $\begin{array}{l}\text { Cd: } 5 \\
\text { Cu: } 1.000 \\
\text { Cr: } 50 \\
\text { Zn: } 5.000 \\
\text { Pb: } 50\end{array}$ \\
\hline Unidad de medición & $\mathrm{ug} / \mathrm{l}$ & $\mathrm{ug} / \mathrm{l}$ \\
\hline $\begin{array}{l}\text { Periodo de promediol } \\
\text { Norma aplicable }\end{array}$ & $\begin{array}{l}24 \mathrm{~h} \\
\text { Decreto 831/93 }\end{array}$ & $\begin{array}{l}24 \mathrm{~h} \\
\text { Decreto } 831 / 93\end{array}$ \\
\hline Periodicidad & \multicolumn{2}{|c|}{$\begin{array}{l}\text { Debe realizarse la determinación antes de su disposición final o } \\
\text { reutilización. }\end{array}$} \\
\hline Destinatario & \multicolumn{2}{|c|}{$\begin{array}{l}\text { Gestión pública (ej.: municipio). Gestión privada (empresa de } \\
\text { fundición). }\end{array}$} \\
\hline $\begin{array}{l}\text { Fuentes y Carencias } \\
\text { de Datos }\end{array}$ & \multicolumn{2}{|c|}{$\begin{array}{l}\text { Se cuenta con estudios locales antecedentes con valores para el } \\
\text { seguimiento de estos compuestos. }\end{array}$} \\
\hline Fortalezas y Debilidades & \multicolumn{2}{|c|}{$\begin{array}{l}\text { F: Los indicadores propuestos pueden utilizarse como } \\
\text { herramienta de gestión para la toma de decisiones en la } \\
\text { reutilización de las arenas. D: pueden presentarse dificultades } \\
\text { técnicas y económicas para su aplicación. }\end{array}$} \\
\hline
\end{tabular}

babilísticos) de contaminación del agua subterránea" en S. Foster y R. Hirata ${ }^{[30 \text { y 31] } 1998 .}$

\subsection{ICA para aspectos ambientales que impactan el recurso agua}

A partir de la descripción realizada en el apartado 2 sobre las características que definen los efluentes líquidos y debido al análisis realizado en el apartado 5.2, se cree que la disposición de los RAF es el principal aspecto ambiental preocupante en relación al recurso hídrico subterráneo, agravándose debido a cómo son actualmente dispuestos porque las áreas utilizadas como relleno para estas arenas no están estructuralmente preparadas para la protección del recurso hídrico subterráneo.

Ante esta situación, y dadas las limitaciones antes mencionadas, se ha pensado en un indicador que considere las características intrínsecas del medio, como es la vulnerabilidad del acuífero (S. Foster ${ }^{[31]}$ (2002)) en los diferentes sectores destinados a relleno y la cantidad de residuo allí depositado.

Es decir, si la vulnerabilidad es la consecuencia de las características naturales de los estratos que separan al acuífero de la superficie del suelo ${ }^{[31]}$, se la puede vincular con la cantidad de residuo depositado, como un primer estado de alerta que amerite indagar a posteriori sobre el peligro de contaminación a través de la metodología antes mencionada (S. Foster ${ }^{[31]}$ ). 
INDUSTRIAS DE FUNDICIÓN: ASPECTOS AMBIENTALES E INDICADORES DE CONDICIÓN AMBIENTAL FOUNDRY INDUSTRIES: ENVIRONMENTAL ASPECTS AND ENVIRONMENTAL CONDITION INDICATORS

Tabla V. Determinación de ICA para residuos sólidos de fundiciones

Table V. ECl determination for foundry solid wastes

\begin{tabular}{|c|c|c|c|}
\hline Aspecto ambiental/ & & Arenas de descarte/Suelo & \\
\hline Indicador & $\begin{array}{l}\text { Sitios con disposición final } \\
\text { de arenas en funcionamiento }\end{array}$ & $\begin{array}{l}\text { Residuos de arena de } \\
\text { fundición }\end{array}$ & $\begin{array}{l}\text { Capacidad total de } \\
\text { almacenamiento }\end{array}$ \\
\hline Descripción & $\begin{array}{l}\text { Informa sobre el aumento o } \\
\text { disminución de los sitios con } \\
\text { disposición final de RAF en } \\
\text { un año }\end{array}$ & $\begin{array}{l}\text { Informa sobre la cantidad } \\
\text { de residuo de arena } \\
\text { generada en cada empresa } \\
\text { de acuerdo al material que } \\
\text { funde }\end{array}$ & $\begin{array}{l}\text { Informa sobre el espacio total } \\
\text { disponible, expresado en } \\
\text { volumen, de los sitios con } \\
\text { disposición de RAF en } \\
\text { funcionamiento. }\end{array}$ \\
\hline Valor de base (Vb) & 15 sitios (año 2008) & $\begin{array}{l}\text { Coeficiente de generación } \\
\text { de residuo de arena: } \\
1 \mathrm{t} \text { de arena }=1 \mathrm{t} \text { de } \\
\mathrm{Fe} ; \mathrm{C}_{\mathrm{Fe}}=1: 1 \\
4 \mathrm{t} \text { de arena }=1 \mathrm{t} \mathrm{Al} ; \mathrm{C}_{\mathrm{Al}}=4: 1\end{array}$ & $\begin{array}{l}\text { Es la capacidad del sitio } \\
\text { en la primera aplicación } \\
\text { del indicador (se contempla } \\
\text { de esta forma la } \\
\text { incorporación de valores de } \\
\text { base de nuevos sitios al } \\
\text { indicador). }\end{array}$ \\
\hline Valor absoluto & $\mathrm{N}^{0}$ de sitios & t de RAF & $M^{3}$ \\
\hline Valor del indicador & $\begin{array}{l}\text { Positivo = aumento de sitios } \\
\text { Negativo = disminución } \\
\text { de sitios } \\
\text { Cero = mantenimiento }\end{array}$ & $\begin{array}{l}\text { Toneladas de residuos de } \\
\text { arena de } \mathrm{Al} \text { y } \mathrm{Fe} \text {. }\end{array}$ & $\begin{array}{l}\text { Positivo = mayor capacidad } \\
\text { (puede estar dada por nuevos } \\
\text { sitios, limpieza de sitios). } \\
\text { Negativo = disminución de la } \\
\text { capacidad por acumulación } \\
\text { de RAF } \\
\text { Cero = no hay variación. }\end{array}$ \\
\hline Fórmula & $\begin{array}{l}S F=S a-V b \\
\text { Donde } S F=\text { variación del } \\
\text { número de sitios; } \mathrm{Sa}=\text { número } \\
\text { de sitios actuales; } \mathrm{Vb}=\text { valor } \\
\text { de base }\end{array}$ & $\begin{array}{l}R A F_{t}=\sum P_{r} \times C_{r} \\
\text { Donde } R A F_{t}=\text { arena } \\
\text { desechada en el total de las } \\
\text { fundiciones; } P_{r}=\text { producción } \\
\text { del rubro; } C_{r}=\text { Coef. del rubro }\end{array}$ & $\begin{array}{l}C s f_{i}=C_{i}-C_{v b} \\
\text { Donde } \text { Csf }_{i}=\text { Variación de la } \\
\text { capacidad del sitio } \mathrm{i} ; \\
\mathrm{C}_{\mathrm{i}}=\text { capacidad actual } \\
\mathrm{Cvb}_{\mathrm{i}}=\text { valor de base de } \\
\text { capacidad del sitio i }\end{array}$ \\
\hline Valor relativo & $\begin{array}{l}\% \text { de variación de sitios en } \\
\text { funcionamiento respecto de } \\
\text { un valor de referencia. }\end{array}$ & $\begin{array}{l}\% \text { de arenas del rubro } X \\
\text { respecto al total }\end{array}$ & $\begin{array}{l}\text { \% de variación de la } \\
\text { capacidad de los sitios de } \\
\text { disposición de residuos } \\
\text { respecto del valor de } \\
\text { referencia }\end{array}$ \\
\hline Fórmula & $\begin{array}{l}S F r=\frac{S a-V b}{V b} \times 100 \\
\text { Donde } S F r=\text { valor relativo } \\
\text { de los sitios en } \\
\text { funcionamiento; } S a=\text { número } \\
\text { de sitios actuales; } V b=\text { valor } \\
\text { de base. }\end{array}$ & $\begin{array}{l}R A \mathrm{Re}_{r}=\frac{P_{r} \times C_{r}}{R A F} \times 100 \\
\text { Donde RARel }=\text { residuo } \\
\text { de arena relativo por rubro; } \\
r=\text { rubro considerado }(\mathrm{Al} ; \mathrm{Fe})\end{array}$ & $\begin{array}{l}C s f=\sum \frac{C_{i}-C v b_{i}}{C v b_{s}} \times 100 \\
\text { Donde Csf }=\text { valor relativo de } \\
\text { la capacidad de los sitios en } \\
\text { funcionamiento; } C_{i}=\text { Capacidad } \\
\text { del sitio } i ; C v b_{i}=\text { valor de } \\
\text { base de capacidad del sitio } i ; \\
C v b_{s}=\text { valor de base de } \\
\text { capacidad de todos los sitios }\end{array}$ \\
\hline
\end{tabular}


Tabla V (continuación). Determinación de ICA para residuos sólidos de fundiciones

Table V (continuation). ECI determination for foundry solid wastes

\begin{tabular}{|c|c|c|c|}
\hline $\begin{array}{l}\text { Aspecto ambiental/ } \\
\text { Recurso }\end{array}$ & & Arenas de descarte/Suelo & \\
\hline Indicador & $\begin{array}{l}\text { Sitios con disposición final } \\
\text { de arenas en funcionamiento }\end{array}$ & $\begin{array}{l}\text { Residuos de arena de } \\
\text { fundición }\end{array}$ & $\begin{array}{l}\text { Capacidad total de } \\
\text { almacenamiento }\end{array}$ \\
\hline Periodicidad & anual & anual & anual \\
\hline Destinatario & $\begin{array}{l}\text { Gestión pública } \\
\text { (ej.: municipio). }\end{array}$ & $\begin{array}{l}\text { Gestión privada } \\
\text { (empresa de fundición). }\end{array}$ & Gestión pública y privada \\
\hline $\begin{array}{l}\text { Fuentes y Carencias } \\
\text { de Datos }\end{array}$ & $\begin{array}{l}\text { Se cuenta con antecedentes } \\
\text { sobre la dinámica de sitios } \\
\text { de disposición final de RAF }\end{array}$ & $\begin{array}{l}\text { Se conoce la producción } \\
\text { de las empresas. }\end{array}$ & $\begin{array}{l}\text { Se conoce la ubicación y } \\
\text { capacidad de los sitios } \\
\text { actuales con disposición } \\
\text { de RAF. }\end{array}$ \\
\hline $\begin{array}{l}\text { Fortalezas y } \\
\text { Debilidades }\end{array}$ & $\begin{array}{l}\text { F: El monitoreo sólo requiere } \\
\text { de un observador. } \\
\text { D: No permite inferir la } \\
\text { cantidad de RAF depositado. }\end{array}$ & $\begin{array}{l}\text { F: no requiere de ningún } \\
\text { costo ya que la empresa } \\
\text { es la propia fuente de datos } \\
\text { y el cálculo es simple. } \\
\text { D: sólo se conoce el coef. } \\
\text { de Al y Fe }\end{array}$ & $\begin{array}{l}\text { F: permite valorar el impacto } \\
\text { ambiental consecuente de la } \\
\text { acumulación de RAF en } \\
\text { inmediaciones de las } \\
\text { industrias. } \\
\text { D: No permite saber la } \\
\text { relación del volumen } \\
\text { acumulado con la carga } \\
\text { contaminante. }\end{array}$ \\
\hline
\end{tabular}

En vista de lo expresado recientemente, y de acuerdo al conocimiento que se tiene de la operatividad de las industrias locales en cuanto a su tamaño, producción y distribución en el área de estudio ${ }^{[9]}$, no se proponen ICA para el monitoreo de los efluentes líquidos de estas industrias, aunque sí para la posibilidad de contaminación del recurso hídrico por disposición de RAF y RIF (Tabla VI).

\section{CONCLUSIONES}

En el trabajo se proponen un total de quince ICA, nueve indicadores para el monitoreo del estado o condición del recurso aire, cinco para el recurso suelo, y uno para el recurso agua.

- Los indicadores en su conjunto permiten determinar la situación ambiental en el entorno de industrias de fundición porque aportan datos técnicos de emisiones de la actividad sobre los recursos que la sustentan, lo que hasta el momento se desconoce.
- La adaptación de metodología, para la construcción de indicadores, aplicada al caso particular de las industrias de fundición permite que en cada momento metodológico se tomen decisiones tendentes a seleccionar los aspectos ambientales cuyas emisiones contaminantes son de importancia, pudiendo replicarse este antecedente metodológico en otros procesos industriales y/o para otras situaciones regionales.

- Los indicadores que representan el estado o condición del recurso aire contemplan en su criterio de selección el cuidado de la salud y de los efectos adversos en el medio ambiente, debido a que en la metodología se utiliza legislación de referencia nacional e internacional que se establece según estos criterios. Por consiguiente, son representativos de los temas ambientales prioritarios en estos niveles. Asimismo las frecuencias de medición son acordes a los programas de control y monitoreo aplicados en estas industrias y aprobados por el organismo provincial de aplicación (OPDS).

- La existencia de un trabajo antecedente, de relevamiento de los sitios de disposición final de residuos de industrias de fundición, facilitó la crea- 
INDUSTRIAS DE FUNDICIÓN: ASPECTOS AMBIENTALES E INDICADORES DE CONDICIÓN AMBIENTAL FOUNDRY INDUSTRIES: ENVIRONMENTAL ASPECTS AND ENVIRONMENTAL CONDITION INDICATORS

Tabla VI. Determinación de ICA para lixiviados de RAF y RIF

Table V. ECI determination for Waste Foundry Sand (WFS) and Foundry Industry Wastes (FIW)

Aspecto ambiental/ Recurso

RAF y RIF / Acuífero

Indicador

Descripción

Valor de la vulnerabilidad

Valor del indicador

Fórmula

\section{Posibilidad de contaminación del acuífero por lixiviados de RAF y RIF}

Informa sobre el estado de alerta que representa la disposición de residuos en relación al peligro de contaminación del recurso hídrico subterráneo.

medio: 0,35; medio/alto: 0,45; alto: 0,55; alto/extremo: 0,8; extremo: 1 .

Bajo: < 0,3; medio: 0,3 a 0,4; medio/alto: 0,4 a 0,5 ; alto: 0,5 a

0,7; alto extremo: 0,7 a 0,9; extremo: 1

Donde: $A$ = Alerta; $V_{i}=$ Vulnerabilidad (valor de la vulnerabilidad considerada); $\mathrm{P}_{\mathrm{i}}=$ peso o cantidad de residuo en un sitio; $\mathrm{P}_{\mathrm{T}}=$ peso total correspondiente a los residuos de todos los rellenos del área

Periodicidad Anual

Destinatario Gestión pública y privada

Fuentes y Carencias de Datos Existen datos antecedentes de la cantidad de rellenos existentes y del volumen acumulado, además se conoce el valor de la densidad para este tipo de residuo por tanto, es posible obtener el peso para cada sitio de disposición. Como carencia se identifica la falta de un mapa completo de vulnerabilidades de la zona de estudio.

\section{Fortalezas y Debilidades}

F: es un indicador simple y económico ya que sólo precisa recorrer los sitios y estimar el peso de los residuos acumulados. Como herramienta de gestión permite conocer en qué medida la acumulación de RAF y RIF en los distintos sitios incrementa o disminuye el estado de alerta en referencia al peligro de contaminación del acuífero en el área.

D: el estado de alerta a la contaminación del acuífero respecto de los RAF y RIF esta sólo en función de la vulnerabilidad del medio y de la distribución de la cantidad de los residuos en ellos; no se consideran variables como el tiempo de permanencia del residuo o la carga contaminante.

ción de indicadores que versan sobre los cambios en la disposición de estos residuos partiendo de una situación conocida. Los indicadores propues- tos tienen la ventaja de ser muy simples en su cálculo y accesibles para la obtención del dato. De la lectura de los indicadores se obtiene infor- 
mación valiosa sobre la evolución de los sitios de disposición de residuos de estas industrias en tres aspectos que se relacionan: en el avance o disminución de basurales dentro de la ciudad (los sitios se encuentran dentro del área urbana), en la relación que hay entre el crecimiento productivo de la industria y la generación de RAF y en los cambios de las capacidades de almacenamiento de los sitios preexistentes, lo que puede anticipar la aparición de nuevos sitios.

- Se concluye además, que el recurso hídrico puede ser afectado por la lixiviación de los RAF y RIF (residuos de industrias de fundición). Se ha logrado un indicador conformado por la sumatoria de subindicadores o indicadores parciales (términos) que responden a los diferentes sectores con su vulnerabilidad intrínseca conocida, afectados por la disposición de residuos en diferente intensidad en cuanto a la carga (peso), y que permite una aproximación de alerta e indica la urgencia de estudios de detalle que estimen el peligro de contaminación del acuífero.

- Si bien el indicador general presentará las variaciones considerando la ponderación de los diferentes aportes (términos) es de destacar la importancia de evaluar en forma particular cada término (subindicador o indicador parcial) y considerar su variación, la cual ofrecerá información de la urgencia de estudios de mayor detalle en cada sector.

- Para finalizar, importa destacar que los ICA propuestos sirven de base de información para la gestión tanto pública como privada. Si estos sectores utilizan el mismo indicador se facilita el diálogo entre las partes, y la búsqueda de solución conjunta del problema que monitorea, y se alcanza el fin principal por el cual se construyen estos indicadores: que sean una herramienta en la toma de decisiones.

\section{REFERENCIAS}

[1] ISO 14032, Gestión ambiental. Ejemplos de evaluación del rendimiento ambiental (ERA), 1999.

[2] World Steel Association (worldsteel), World Steel Sustainability Indicator Methodology. Disponible en http://www.worldsteel.org/dms/ internetDocumentList/downloads/steel-bytopic/Indicator-Methodology/document/ IndicatorMethodology_rev2011.pdf [15/07/2012]

[3] Acindar Grupo Arcelor Mital, Reporte de Sustentabilidad, 2010, pp. 34-38. Disponible en http://www.acindar.com.ar/reporte10/pdf/reporte.pdf [11/07/2012]

[4] Ministerio de Salud y Ambiente, Iniciativa Latinoamericana y Caribeña para el Desarrollo Sostenible-Indicadores de Seguimiento: SAyDS $1^{\mathrm{a}}$ ed., Buenos Aires, Argentina, 2006, pp. 1 72.

[5] Programa de las Naciones Unidas para el Medio Ambiente, GEO América Latina y el Caribe, Perspectivas del Medio Ambiente, PNUMA, México, D.F., 2003, pp. 254-279.

[6] Secretaria de Ambiente y Desarrollo Sustentable de la Nación, Primer Compendio de Estadísticas Ambientales: República Argentina, SAyDS $1^{\mathrm{a}}$ ed., Buenos Aires, Argentina, 2008, pp. 24-34, 185-373.

[7] Secretaría de Ambiente y Desarrollo Sustentable de la Nación, GEO Argentina 2004, Perspectivas del Medio Ambiente de la Argentina, SAyDS y PNUMA, Buenos Aires, Argentina, 2006, pp. 24-25.

[8] B. Sosa, R. Banda-Noriega y M. Guerrero, IVoㅡ Jornadas ASAUEE, Nuevos escenarios globales y Alternativas para un Desarrollo Local Sostenible. Horacio A. Feinstein (ed.), Buenos Aires, Argentina, 2009, p. 59.

[9] B. Sosa, R. Banda-Noriega y M. Guerrero, Rev. Gestión y Ambiente. Industria, emisiones gaseosas y gestión ambiental urbana: Diagnóstico de las condiciones operativas de las fundiciones en Tandil, Argentina. Estado: enviado $(12 / 2011)$

[10] Secretaría de Ciencia Tecnología e Innovación Productiva, Industria Manufacturera Argentina, Análisis del comercio exterior según su intensidad tecnológica, Buenos Aires, Argentina, 2007, p. 15 Disponible en http://www.mincyt.gov.ar/multimedia/archivo/archivos/analisis_del_comercio_exterior_segun_intensidad_tecnologica.pdf [27/07/2012]

[11] Organización para el Desempeño Empresarial Sostenible (ODES), Diagnóstico Integral Sector de Fundición en el Departamento del Atlántico, Barranquilla, España, 2005, pp. 36-37, Disponible en http://www.crautonoma.gov.co/ documentos/odes/1InformeDiagnosticoSectorial.pdf [30/07/2012].

[12] ISO 14031, Evaluación del Desempeño Ambiental - Directrices, Traducción Oficial, Ginebra, Suiza, 1999, p.1.

[13] Consejo Nacional de Producción Limpia, Guía para el control y prevención de la contaminación industrial: rubro fundiciones. Región Metropolitana, Santiago de Chile, 1998, pp. 12 17. Disponible en http://www.sofofa.cl/ 
INDUSTRIAS DE FUNDICIÓN: ASPECTOS AMBIENTALES E INDICADORES DE CONDICIÓN AMBIENTAL FOUNDRY INDUSTRIES: ENVIRONMENTAL ASPECTS AND ENVIRONMENTAL CONDITION INDICATORS

ambiente/documentos/Rubros\%20Fundiciones.pdf [30/07/2012]

[14] Documento de referencia de Mejores Técnicas Disponibles en la Industria de Procesos de Metales no Férreos, Centro de Publicaciones Secretaría General Técnica Ministerio de Medio Ambiente, España, 2005, pp. 53-188 y 269-327.

[15] J. Barr, Especies, Espacios y Riesgos, Raúl Marcó del Pont Lalli (Ed.), Instituto Nacional de Ecología (INE- SEMARNAT), México, 2006, pp. 181-194.

[16] R. Quiroga-Martinez, Guía metodológica para desarrollar indicadores ambientales y de desarrollo sostenible en países de América Latina y el Caribe, CEPAL-Serie Manuales Nº 61, 2009, pp. 6670 y 104.

[17] Eusko Jaurlaritza- Gobierno Vasco, Guías Técnicas para la Medición, Estimación y Cálculo de las Emisiones al Aire I, IHOBE, 2008, pp. 23-25.

[18] Eusko Jaurlaritza- Gobierno Vasco, Guías Técnicas para la Medición, Estimación y Cálculo de las Emisiones al Aire 7, IHOBE, 2008, pp. 23-26.

[19] Eusko Jaurlaritza- Gobierno Vasco, Guías Técnicas para la Medición, Estimación y Cálculo de las Emisiones al Aire 9, IHOBE, 2008, pp. 22-24, 34-38.

[20] Marco Regulatorio de la Provincia de Buenos Aires. Ley № 5965, Decreto Reglamentario 3395/1996, Resolución 242/1997, disponible en: http://www.opds.gba.gov.ar/index.php/leyes/resumen [30/07/2012]

[21] United States Environmental Protection Agency (EPA), National ambient air quality standards. Disponible en $h t t p: / / w w w . e p a . g o v /$ air/criteria.html [31/07/2012]

[22] Organización Mundial de la Salud (OMS), Guías de calidad del aire de la OMS relativas al material particulado, el ozono, el dióxido de nitrógeno y el dióxido de azufre. Actualización mundial
2005. Disponible en http://whqlibdoc.who. int/hq/2006/WHO_SDE_PHE_OEH_06.02_spa. pdf [26/07/2012]

[23] Organismo Provincial para el Desarrollo Sostenible. Marco Regulatorio de la Provincia de Buenos Aires, Ley Provincial 11720 su Decreto Reglamentario 806/97, disponible en: http://www.opds.gba.gov.ar/index.php/leyes/resumen [30/07/2012]

[24] R.E. Miguel, V.A. Ruiz de Galarreta y R.B. Banda-Noriega, Tesis de Maestría, Facultad Regional La Plata, Universidad Tecnológica Nacional, 2009.

[25] A. Deng, J. Environ. Pollut. 38-4 (2009) 425-443.

[26] R.S. Dungan y N.H. Dees, J. Environ. Manage, 90 (2009) 539-548.

[27] R.B. Banda-Noriega, R.E. Miguel, R.G. Marcozzi y J.D. Sota, 15ำ Congresso de Fundição da ABIFA, São Paulo, Brazil, 2011, disponible en CD.

[28] Secretaría de Medio Ambiente y Recursos Renovables. Argentina, Ley Nacional de Residuos Peligrosos 24051, Decreto 831/93, Actualizado 2010, http://www.ambiente.gov. ar/? aplicacion=normativa \&IdNorma $=538 \& I d S e c c i$ on $=0$ [19/07/2012]

[29] United States Environmental Protection Agency (EPA), Wastes-Hazardous Waste-Test Methods 846, Method 1311 Toxicity Characteristic Leaching Proceduresw, Disponible en http://www.epa.gov/osw/hazard/testmethods/sw846/pdfs/1311.pdf [15/07/2012]

[30] S. Foster y R. Hirata, Determinación del Riesgo de Contaminación de Aguas Subterráneas, CEPIS, (OMS, OPS), Lima, Perú, 1998. Disponible en http://www.busde.paho.org/eswww/ fulltext/repind46/riego/riego.html [31/07/2012]

[31] S. Foster, R. Hirata, D. Gomes, M. D’Elia y M. Paris, Protección de la calidad del agua subterránea. Ed. Mundi Prensa, Madrid, España, 2003, pp. 18-19, 88. 